\title{
HEURÍSTICA DA ANCORAGEM E AJUSTAMENTO COMO INFLUENCIADORA DA INTENÇÃO DE COMPRA DE TECNOLOGIA DA INFORMAÇÃO VERDE
}

\author{
Ana Amélia Zwicker \\ Doutoranda em Administração \\ Universidade Federal de Santa Maria \\ Santa Maria - Rio Grande do Sul - Brasil \\ ana.ameliamz@gmail.com \\ Mauri Leodir Löbler \\ Doutor em Administração \\ Universidade Federal de Santa Maria \\ Santa Maria - Rio Grande do Sul - Brasil \\ mllobler@gmail.com
}

\section{RESUMO}

A Tecnologia da Informação encontra-se arraigada no escopo do desenvolvimento em uma escala global. Em virtude disso, abordar o consumo na perspectiva da Tecnologia da Informação Verde (TI Verde) assume grande relevância no enfrentamento dos desafios para consecução da sustentabilidade ambiental. Assim, o objetivo deste estudo é verificar o efeito da ancoragem na intenção de compra dos consumidores de TI Verde. Em um delineamento complexo, um quase experimento foi aplicado em laboratório, com alunos de Engenharia Sanitária e Ambiental, Administração e Sistemas da Informação de uma Universidade Federal, totalizando 170 respondentes. A âncora utilizada foi um vídeo informacional sobre lixo eletrônico previamente exposto à fase de instrumentalização da pesquisa. Para o experimento foram elaborados dois tipos de anúncios $(\mathrm{A}=$ com argumento ecológico (AE); $\mathrm{B}=$ sem argumento ecológico), utilizando-se informações reais de comercialização de um notebook. A partir das análises quantitativa e qualitativa, os resultados indicam mais um discurso sustentável do que uma intenção de compra propriamente dita: os alunos, em geral, mencionaram fortemente o $\mathrm{AE}$ como aspecto do produto importante em suas opiniões, porém, isto não se refletiu na intenção de compra. Contudo, as evidências sugerem que o conhecimento dos respondentes, aliado à ancoragem, interferem na intenção de compra dos sujeitos em relação à TI Verde, enfatizando a necessidade de estudos mais aprofundados em relação aos efeitos de diferentes contextos de aplicação nos resultados. Assim, a contribuição desta pesquisa está na introdução de ancoragem como uma heurística cognitiva para a área de consumo de TI Verde.

Palavras-chave: Tecnologia da Informação Verde (TI Verde). Consumo de Tecnologia da Informação. Sustentabilidade. Heurística da Ancoragem e Ajustamento

\section{HEURISTICS OF ANCHORAGE AND ADJUSTMENT AS INFLUENCING FACTORES TO THE INTENTION TO BUY GREEN INFORMATION TECHNOLOGY}

\begin{abstract}
Information Technology is rooted in the scope of development on a global scale. As a result, addressing consumption from the perspective of Green Information Technology (Green IT) is of great relevance in meeting the challenges of achieving environmental sustainability. Thus, the objective of this study is to verify the effect of anchoring in the purchase intention of Green IT consumers. In a complex design, a near experiment was applied in the laboratory, with students of Sanitary and Environmental Engineering, Administration and Information Systems of a Federal University, totaling 170 respondents. The anchor used was an informational video about electronic waste previously exposed to the phase of instrumentalization of the research. For the experiment two types of ads were produced $(\mathrm{A}=$ with ecological argument $(\mathrm{AE}), \mathrm{B}=$ no ecological argument), using real information of commercialization of a notebook. From the quantitative and qualitative analyzes, the results indicate a more sustainable discourse than a purchasing intention per se: students, in general, strongly mentioned EC as an important product aspect in their opinions, but this was not reflected in the buy intention. However, the evidence suggests that the knowledge of the respondents, together with the anchorage, interfere with the subjects' intention to buy Green IT, emphasizing the need for more in-depth studies regarding the effects of different application contexts on the results. Thus, the contribution of this research is in the introduction of anchorage as a cognitive heuristic for the area of Green IT consumption.
\end{abstract}

Key words: Green Information Technology (Green IT). Information Technology Consumption. Sustainability. Anchoring-and-Adjustment Heuristic. 


\section{INTRODUÇÃO}

Tecnologia da Informação Verde (TI Verde) trata-se da adoção de indivíduos e de organizações de práticas ambientalmente sustentáveis em torno do uso da tecnologia da informação e de seus sistemas associados (Murugesan, 2008). Na esteira deste processo, este trabalho se concentra no comportamento de consumo em nível de indivíduos, abrangendo os temas Sustentabilidade, Tecnologia da Informação (TI) e Processo Decisório, por meio da Heurística da Ancoragem e Ajustamento.

Em referência à mencionada teoria, esta verifica a chance de ocorrência de um evento pela colocação de uma base (âncora) e, então, faz-se um ajuste (Bazerman, 2004). Na Heurística da Ancoragem e Ajustamento, os indivíduos focalizam a atenção sobre uma informação recentemente recebida e a usam como referência para fazer uma estimativa ou tomar uma decisão. A âncora é uma informação relevante que está disponível ao tomador de decisão que pode ser inserida na formulação do problema em questão, ou pode ser resultado de uma análise parcial. Assim, a ancoragem refere-se a confiar fortemente em uma peça de informação (a âncora) que aparece antes dos tomadores de decisão fazerem um julgamento (Tversky \& Kahneman, 1974). Para Luppe e Angelo (2010), com a adoção de um referencial, lógico ou não, pode-se direcionar a escolha dos indivíduos.

Conforme Epley e Gilovich (2006), não está claro porque os ajustes (efeitos após colocação da âncora) da Heurística da Ancoragem e Ajustamento tendem a ser insuficientes. Estudos nesta área podem ser realizados em diferentes situações, em nível de decisão empresarial ou de indivíduos, cabe destacar que esta pesquisa concentra-se em nível de intenção de compra de um artefato de uso pessoal de tecnologia da informação.

Segundo Schimidt, Schmidtchen, Erek, Kolbe e Zarnekow (2010), dentre as facetas da TI Verde que necessitam ser aprofundadas, destaca-se a relacionada ao consumidor, tendo em vista a importância do seu papel, por meio de seu poder de escolha, para a efetiva implementação de uma TI sustentável pelas empresas. Atualmente, as crianças já nascem conectadas a produtos eletrônicos; adultos e idosos estão cada vez mais se inserindo no contexto tecnológico; estudantes e professores possuem ferramentas de TI como apoio à aprendizagem. Mas uma indagação surge: a Tecnologia da Informação Verde é considerada no processo de consumo destes produtos e serviços? Pessoas que possuem um discurso ambiental politicamente correto aplicam-no na intenção de compra de um dispositivo de tecnologia da informação?

Analisando-se os padrões atuais de consumo, é necessário desenvolver a consciência ecológica do consumidor e incentivar padrões responsáveis de consumo. Porém, estudos sobre comportamento sustentável por consumidores finais se baseiam em muitos estudos do tipo survey, onde os indivíduos são questionados sobre suas percepções, intenções ou comportamentos, sem efetivamente se posicionarem sobre um produto com argumentos ecológicos. Nesse trabalho, buscou-se compreender o comportamento do indivíduo numa situação de compra de TI, variando um produto com e sem argumento ecológico e inserindo uma variável interveniente no processo, uma informação, que se denominou âncora, para confirmar, ou não, se o comportamento ecologicamente correto é algo internalizado ou apenas aparece após um estímulo nessa direção. Complementarmente, esta pesquisa possibilita verificar a influência do marketing verde nas decisões de consumo.

Além disso, com relação ao consumo verde e à TI Verde, não foi encontrado trabalho que referenciasse a ancoragem como estratégia de pesquisa. Face ao exposto, a pergunta de pesquisa é: os consumidores levam em consideração aspectos de sustentabilidade na aquisição de Tecnologia de Informação ou modificam pelo efeito âncora, manipulado numa pesquisa experimental?

Assim, o objetivo deste estudo é verificar o efeito da ancoragem na intenção de compra dos consumidores de Tecnologia da Informação Verde. 


\section{TECNOLOGIA DA INFORMAÇÃO VERDE - TI VERDE}

Segundo Elliot e Binney (2008), TI Verde envolve design, produção, operação e descarte de produtos e serviços de tecnologia de informação, apoiados nas TIs de uma forma que não seja prejudicial e que seja benéfica para o ambiente durante o curso de toda sua vida. Para Jayo e Valente (2010), estão na agenda de decisões desta área: desenvolvimento de produtos com maior vida útil e maior eficiência energética, mudanças nos hábitos de consumo, visando ao descarte consciente, implementação de regulamentações que obriguem a indústria a reduzir a toxicidade e implementar programas de reciclagem de seus próprios produtos. Murugesan (2008) afirma que muitos fabricantes de computadores estão no processo de fazer equipamentos verdes usando materiais não-tóxicos que consomem menos energia elétrica e são facilmente remontados, sendo altamente atualizáveis, prolongando assim a sua vida útil. No que diz respeito aos hábitos de consumo, na perspectiva dos clientes, o consumo de energia durante a operação e a disposição de um computador no fim de seu ciclo de vida podem ser considerados como atributos de TI Verde (Schmidt et al., 2010).

O setor de TI e seus usuários devem desenvolver uma atitude positiva em relação às preocupações ambientais e adotar políticas e práticas voltadas para o futuro e que respeitem o meio ambiente (Murugesan, 2008). Segundo Koo e Chung (2014), a revolução da TI Verde parece estar destinada a transformar a indústria moderna, o comportamento do consumidor e a civilização constantemente e prevê progressos sociais de forma mais sensata na sociedade moderna.

Berkhout e Hertin (2001) dividem os impactos de TI no meio ambiente em três categorias:

a) de primeira ordem: impacto direto sobre o ambiente por meio da produção, logística, uso e descarte;

b) de segunda ordem: impactos indiretos ao ambiente relacionados aos efeitos das TIs na estrutura econômica, sistemas de produção e distribuição;

c) de terceira ordem: impactos indiretos ao ambiente por meio do estímulo do consumo verde e crescimento econômico das TIs, e pelo impacto no estilo de vida e no sistema de valores.

Este artigo alcança os três níveis acima mencionados: a primeira dimensão, pois referencia o uso e descarte ambientalmente corretos de um computador; a terceira dimensão, porque verifica as atitudes dos sujeitos em relação aos anúncios de TI e à ancoragem de vídeo com propósito de direcionar uma intenção de compra mais sustentável, sendo que estas atitudes podem interferir na estrutura econômica, sistemas de produção e distribuição (segunda ordem).

Salles, Alves, Dolci e Lunardi (2016) ressaltam que colocar em prática a TI Verde demanda mudanças em termos de tecnologias (melhoria da infraestrutura de TI e de negócios para torná-las ambientalmente corretas) e de comportamento - por meio do agir de maneira ambientalmente responsável. Segundo Ebreo e Vining (2001), mesmo que avanços tecnológicos para conservação dos recursos e para a redução da poluição tenham ocorrido ou venham a ocorrer, as ações no âmbito individual ou doméstico precisam ser consideradas para redução dos problemas de degradação ambiental do planeta.

Com relação ao consumo de TI Verde, a Electronic Product Environmental Assessment Tool (Epeat) é um sistema de classificação ambiental global que ajuda compradores na identificação de computadores mais verdes e outros eletrônicos, apoiando os esforços de aquisição verdes de governos, empresas, instituições e consumidores. A Epeat fornece rótulo ecológico a empresas que cumprem uma série de normas ambientais, sendo os critérios classificados como obrigatórios ou optativos. Todos os critérios utilizados são baseados em padrões públicos aprovados pela American National Standards Institute (ANSI), na qual um produto deve atender a todos os exigidos em sua categoria para ser adicionado ao registro. Em seguida, recebe a classificação bronze, prata ou ouro, dependendo de quantos critérios opcionais atingiu: produtos bronze cumpriram todos os critérios obrigatórios, os de classificação prata alcançaram todos os exigidos e pelo menos $50 \%$ dos critérios opcionais, e os com classificação ouro cumpriram todos os itens exigidos e pelo menos $75 \%$ dos 
critérios opcionais (Epeat, 2016). Murugesan (2008) destaca a Epeat como uma das ferramentas para identificação de produtos de TI sustentáveis.

Todos os computadores registrados na Epeat têm níveis reduzidos de cádmio, chumbo e mercúrio para melhor proteger a saúde humana. São mais eficientes em termos energéticos e mais fáceis de atualizar e reciclar. Os fabricantes de produtos com selo da Epeat devem oferecer opções de reciclagem seguras para os produtos quando eles não são mais utilizáveis (Murugesan, 2008).

Considerando o ciclo de vida completo de computadores, as categorias da Epeat englobam: redução/eliminação de materiais ambientalmente sensíveis; seleção de materiais; design para o fim da vida; longevidade do produto; conservação de energia; gestão do fim da vida; desempenho corporativo; embalagem. Este sistema também considera uma variedade de critérios relacionados ao serviço: devolução e reciclagem responsável de produtos, embalagem e baterias, bem como a prestação de garantia estendida e outros serviços que podem reduzir o impacto ambiental. Produtos registrados na Epeat estão de acordo com as mais recentes especificações da Energy Star ${ }^{\circledR}$ e são projetados, fabricados e apoiados para reduzir o teor tóxico e de resíduos sólidos, diminuir as emissões e aumentar a capacidade de reciclagem (Epeat, 2016).

Atualmente, são 43 países registrados na Epeat, dentre os quais o Brasil está inserido. Segundo o site desta associação, toda vez que alguém compra um produto com este registro incentiva os fabricantes a produzir eletrônicos que atendam os critérios ambientais especificados, sendo menos prejudiciais ao meio ambiente (Epeat, 2016).

Nas pesquisas de Schmidt et al. (2010), foram considerados os atributos eliminação (descarte) e eficiência energética para escolha de atributos verdes de computadores, os quais os participantes atentaram mais para o primeiro atributo. Desta forma, neste artigo, foi seguida a mesma linha dos autores, com a utilização de argumento ecológico - apelo verde relacionado ao descarte adequado de um notebook pelo consumidor. Por sua vez, a eficiência energética foi considerada com a inclusão do argumento ecológico - selo verde, por meio da Epeat e da Energy Star ${ }^{\circledR}$. Este último é um programa voluntário da Agência de Proteção Ambiental dos Estados Unidos que ajuda as empresas e os indivíduos a economizar dinheiro e proteger o clima por meio da eficiência energética. Desde o seu início, em 1992, o programa Energy Star tem ajudado a identificar e promover a eficiência energética em produtos, casas e edifícios nos Estados Unidos (Energy Star, 2016).

A prática de TI Verde não é tarefa fácil: há muitos fatores de riscos que devem ser identificados e monitorados numa variedade de aspectos nas ações. Por exemplo, uma prática bemsucedida solicitaria a indivíduos como usuários domésticos, funcionários e gerentes que seguissem normas e políticas ambientais em seu trabalho e em suas casas. Contudo, esses regulamentos e políticas podem frequentemente causar descuidos. As pessoas precisam entender a importância da qualidade ambiental e também obedecer ao conjunto de normas políticas (Chou, 2013). É nesta perspectiva que este estudo se concentra: em âmbito comportamental da TI Verde, procurando associá-lo ao entendimento, à consciência do indivíduo e às suas atitudes.

\section{HEURÍSTICA DA ANCORAGEM E AJUSTAMENTO}

Segundo Mussweiler e Strack (2001), a ancoragem é uma das influências mais notáveis em julgamento e tomada de decisão. No que diz respeito ao processo decisório, o pesquisador Herbert Simon reconheceu a capacidade cognitiva limitada da mente humana, afirmando que o decisor tem restrito conhecimento do número total de consequências de suas decisões, sendo exposto ainda a pressões sociais e pessoais (Löbler, 2005). Assim, métodos heurísticos são utilizados para o processamento da informação dos indivíduos. A expressão heurística tem origem grega e significa descobrir (Russo \& Schoemaker, 1993).

Os pesquisadores Amos Tversky e Daniel Kahneman, baseando-se na ideia de racionalidade limitada de Simon, apresentaram, em 1974, o programa Heurístics and Biases. Os trabalhos destes autores revolucionaram a pesquisa acadêmica no tocante ao julgamento humano (Luppe \& Angelo, 
2010). Estes autores desafiaram o uso de modelos racionais como explicação para os processos de tomada de decisão ao oferecerem três heurísticas: a heurística da representatividade, a heurística da disponibilidade e a heurística da ancoragem e ajustamento (Magalhães, 2013).

Em suas primeiras pesquisas, Tversky e Kahneman (1974) pediram que os participantes estimassem a porcentagem de países africanos nas Nações Unidas, a partir de um número aleatório de uma rodada de roleta. Posteriormente, os respondentes precisavam indicar e estimar a quantidade real em comparação (superior ou inferior) ao valor obtido na roleta. Em outro estudo, estes mesmos autores separaram estudantes de ensino médio em dois grupos dando a cada um uma expressão matemática $(8 \times 7 \times 6 \times 5 \times 4 \times 3 \times 2 \times 1$ ou $1 \times 2 \times 3 \times 4 \times 5 \times 6 \times 7 \times 8)$ para resolução em cinco segundos. Nesta situação, a primeira expressão foi julgada maior do que a segunda. Em ambos os casos, os pesquisadores verificaram a ancoragem na disponibilização de informações claramente não informativas (primeiro estudo) e na estimativa de um cálculo incompleto (segundo estudo).

Toda vez que os indivíduos formam uma impressão ou uma imagem sobre um estímulo quando outro estímulo está presente, essas reproduções podem estar sujeitas a efeitos de ancoragem (Esch et al., 2009). A Heurística da Ancoragem e Ajustamento verifica a chance de ocorrência de um evento pela colocação de uma base (âncora) e então se faz um ajuste. O ponto de partida pode ser um precedente histórico, por meio da apresentação de um problema ou uma informação aleatória (Bazerman, 2004).

Conforme Strack e Mussweiller (1997), a âncora age como se fosse uma sugestão, fazendo com que informações consistentes com a âncora estejam mais disponíveis na memória por meio de mecanismos de preparação ou então por meio de uma pesquisa externa que é viesada pela presença de uma referência. Assim, para um ponto de referência se tornar uma âncora, é preciso haver compatibilidade com uma tarefa focal.

Efeitos de ancoragem são verificados em vários domínios nos estudos de julgamento: questões de conhecimento geral, avaliações de loterias e jogos, estimativas de risco e incerteza, avaliação de preços de imóveis, avaliações do desempenho futuro, efeitos de múltiplas âncoras no julgamento individual e em grupo, avaliações de probabilidade com auditores profissionais, negociações, além de aplicações no mercado de consumo (Mussweiler \& Strack, 2001). Predominantemente, verifica-se nos estudos uma estimativa numérica como âncora, porém, neste trabalho, a base foi um vídeo informacional.

Segundo Luppe e Angelo (2010), há pouca literatura que aborda os vieses da ancoragem atrelados ao processo de escolha em decisões de consumo. A título de demonstração, a Figura 1 expõe alguns estudos que associam ancoragem e consumo, sendo que a pesquisa sobre o tema não foi exaustiva. Especialmente, com relação ao consumo verde e à TI Verde, não foi encontrado trabalho que referenciasse a ancoragem como estratégia de pesquisa.

\begin{tabular}{|c|l|}
\hline Autores & \multicolumn{1}{c|}{ Breve Descrição } \\
\hline Yadav (1994) & $\begin{array}{l}\text { Avaliação de dois pacotes de produtos: computador, impressora, mesa de computador; cama, } \\
\text { cômoda, armário. Os sujeitos analisam os itens por ordem decrescente de importância por meio } \\
\text { de ajustes insuficientes para cima ou para baixo. }\end{array}$ \\
\hline $\begin{array}{c}\text { Wansink, Kent } \\
\text { e Hoch (1998) }\end{array}$ & $\begin{array}{l}\text { Decisão dos consumidores sobre quantos itens comprar em um ponto de venda. Promoções } \\
\text { baseadas em ancoragem podem aumentar a venda de produtos. }\end{array}$ \\
\hline $\begin{array}{c}\text { Donoho } \\
(2003)\end{array}$ & $\begin{array}{l}\text { Experimento com exposição de vídeo sobre eletrônicos, sendo o de maior preço com a indicação } \\
\text { da âncora top de linha. A âncora funciona para a escolha de produtos mais caros. }\end{array}$ \\
\hline $\begin{array}{c}\text { Luppe e } \\
\text { Angelo (2010) }\end{array}$ & $\begin{array}{l}\text { Ancoragem no processo decisório dos consumidores em relação à percepção e à estimação dos } \\
\text { preços de produtos e serviços. Os referenciais com valores menores ancoram mais fortemente a } \\
\text { opinião dos consumidores. }\end{array}$ \\
\hline $\begin{array}{c}\text { Bezerra e } \\
\text { Leone (2013) }\end{array}$ & $\begin{array}{l}\text { Efeito ancoragem em estimativas de preços para produtos da cesta básica. As âncoras } \\
\text { influenciaram as estimativas dos julgadores, impulsionando seus julgamentos para mais ou para } \\
\text { menos, de acordo com a predição inicial a que os indivíduos estiveram expostos. }\end{array}$ \\
\hline $\begin{array}{c}\text { Gagarina e } \\
\text { Pikturniene } \\
(2015)\end{array}$ & $\begin{array}{l}\text { Efeito do perfume ambiental em heurísticas de tomada de decisão, especialmente quando decisões } \\
\text { arriscadas e percepções de risco estão envolvidas. }\end{array}$ \\
\hline Pereira et al. & Efeitos na precificação de produtos similares, porém de marcas distintas, tendo por base a \\
\hline
\end{tabular}

Revista de Gestão Social e Ambiental - RGSA, São Paulo, v. 12, n. 2, p. 39-54, maio/ago. 2018. 


\begin{tabular}{|c|l|}
\hline (2015) & atribuição de âncoras de decisão. \\
\hline $\begin{array}{c}\text { Brunner et al. } \\
(2016)\end{array}$ & $\begin{array}{l}\text { Consumidores são confrontados com diferentes conotações de designs de produtos simbólicos. Os } \\
\text { resultados mostram que as associações de design de produto são usadas no processamento de } \\
\text { informações dos consumidores como âncora para avaliações de marcas. }\end{array}$ \\
\hline $\begin{array}{c}\text { França et al. } \\
(2017)\end{array}$ & $\begin{array}{l}\text { Processo de precificação de veículos seminovos. Âncoras possuem influência no processo } \\
\text { decisório de precificação de veículos seminovos. }\end{array}$ \\
\hline $\begin{array}{c}\text { Liu e } \\
\text { Karahanna } \\
(2017)\end{array}$ & $\begin{array}{l}\text { Os efeitos do balanço das avaliações de produtos on line sobre a construção de preferências de } \\
\text { atributos. }\end{array}$ \\
\hline
\end{tabular}

Figura 1 - Pesquisas de heurística da ancoragem e ajustamento associados ao consumo

Fonte: Elaborado pelos autores.

Em relação aos estudos dos últimos três anos expostos na Figura 1, foi possível verificar que foram encontrados efeitos de ancoragem nas decisões de consumo dos indivíduos. No entanto, no caso de Gagarina e Pikturniene (2015), nem todas as heurísticas foram igualmente expressas em diferentes condições de tipo e intensidade do aroma. Com base nesse embasamento teórico, este estudo propôs a seguinte hipótese: no anúncio com argumento ecológico, os sujeitos submetidos ao efeito ancoragem apresentam maior intenção de compra (Ic).

\section{MÉTODO DE PESQUISA}

Esta pesquisa caracteriza-se como explicativa e quase-experimental, por meio de um delineamento fatorial 2 X 2 X 3 e between subjects (entre sujeitos). Em termos temporais, realizouse a concepção transversal de análise: no mês de outubro de 2016. Foram utilizados nesta pesquisa grupos independentes e feitas manipulações diretas. Ainda, há a presença do delineamento experimental complexo, que manipula mais de uma variável independente em um único experimento, relacionando-se mais com as condições do mundo real, já que as variáveis independentes não existem isoladamente (Cozby, 2006).

Por meio da construção de dois tipos de anúncios (A e B), o experimento utilizou informações reais de comercialização de um notebook, objeto de tecnologia da informação bastante buscado por consumidores graças à sua mobilidade, consumo de energia e simplicidade em relação a desktops (Techtudo, 2013). No anúncio A (grupo experimental), foi inserido um argumento ecológico (AE), o qual serve para fornecer informações sobre a produção ecologicamente correta de produtos, marcas ou empresas e que tem a intenção de alterar o comportamento ou a atitude do consumidor (Pereira, 2003). O anúncio B fez parte do grupo de controle, não fornecendo informações relacionadas à sustentabilidade ambiental.

Realizou-se uma análise quantitativa por meio do software BioStat e qualitativa com o apoio do software NVivo10. Foi realizado o teste de Shapiro Wilk para verificação da normalidade dos dados quantitativos. Tendo em vista que os resultados apontaram para a não normalidade, foi utilizada a estatística não-paramétrica para testar as diferenças entre os grupos (teste U de Mann Whitney).

Para Engel, Blackwell e Miniard (2000), atitude alude a uma avaliação geral do indivíduo que, na maioria das vezes, exerce importante influência no comportamento do consumidor. A variável intenção de compra assume papel de variável dependente neste estudo, tal como nos estudos de Pereira (2003), Caldeira (2009) e Velter et al. (2009). As variáveis independentes são: anúncio A (com argumento ecológico); anúncio B (sem argumento ecológico) e o efeito ancoragem. A âncora utilizada nesta pesquisa foi um vídeo informacional sobre lixo eletrônico e o ajuste que era esperado relaciona-se a uma maior intenção de compra no que diz respeito a um anúncio com argumento ecológico.

Com o objetivo de analisar a interação entre as variáveis, dois anúncios foram construídos tendo como base dados reais obtidos no mês de junho de 2015 no site da Dell. Esta fabricante é registrada na Epeat, a qual já foi explicada no referencial teórico. A Figura 2 desta seção expõe os 
dois anúncios que foram utilizados na pesquisa, sendo que um faz parte do grupo experimental (à esquerda) e o outro do grupo de controle (à direita), respectivamente.

Os sujeitos selecionados para a pesquisa foram alunos de graduação dos cursos de Engenharia Sanitária e Ambiental, Administração - Noturno e Sistemas da Informação da Universidade Federal de Santa Maria (UFSM). A escolha do público universitário foi devido ao fato de que notebooks fazem parte do cotidiano destes estudantes (Lopez, Silva \& Hernandez, 2013). A definição dos cursos se deu em razão da pesquisa empregar argumento técnico de tecnologia da informação (curso de Sistemas da Informação - SI) e argumento ecológico (curso de Engenharia Sanitária e Ambiental - ESA). Buscou-se, ainda, um curso relativamente neutro (curso de Administração - ADM). O propósito destas escolhas foi o de comparar as opiniões dos alunos relativas aos anúncios, considerando três áreas do conhecimento distintas e, por conseguinte, verificar as diferentes vivências e visões dos acadêmicoss em suas áreas ao analisarem o anúncio. Por isso, o critério de seleção das turmas dos três cursos ocorreu do quinto semestre em diante, pois se acredita que, após este período, os alunos já tenham emergido em um conjunto de valores, conhecimentos, habilidades, atitudes, ideologias e culturas relativos ao ambiente do curso que frequentam.

Antes da realização da pesquisa, foram efetivados dois pré-testes. O pré-teste 1 ocorreu no mês de julho de 2016 com alunos do quinto semestre do curso de Administração Diurno. O préteste 2 ocorreu em setembro de 2016 com alunos do primeiro semestre de Administração Noturno. No total, 53 pessoas participaram destas etapas, sendo que suas respostas não fazem parte da análise da pesquisa propriamente dita. Nos pré-testes, as turmas foram divididas em duas, uma parte assistiu ao vídeo e outra não, sendo os alunos designados aos anúncios e à ancoragem aleatoriamente.

Antes da realização do primeiro pré-teste, foram propostos três tipos de anúncios: um com selo verde, outro com apelo verde e um terceiro sem argumento ecológico. Porém, no primeiro préteste, notou-se que a diferenciação entre as pistas selo verde e apelo verde não foi verificada entre os respondentes. Desta forma, optou-se em unir as duas pistas em um anúncio único, a fim de reforçar e concentrar o argumento ecológico em um tratamento para poder aumentar a diferenciação em relação ao anúncio-controle, acrescentando assim maior poder ao teste, agregando maior validade.

Além disso, a proposta desta etapa omitia marca e preço do notebook, entre outros aspectos. Porém, alguns participantes ficaram confusos com a falta de informações e chegaram a sugerir que fosse realizado um teste para verificar aspectos da área de marketing, analisando elementos não relacionados ao produto, tais como layout do anúncio, cores, entre outros. Desta forma, optou-se por incluir mais atributos em relação ao aparelho, a fim de deixar o anúncio mais próximo da realidade de escolha de um consumidor de tecnologia da informação, agregando fidedignidade.

Assim, no desenvolvimento da tarefa, foram incluídos os critérios considerados relevantes no estudo de Reis (2011): tamanho da tela, peso, dimensão, marca e preço, sendo que alguns critérios mencionados pela autora já constavam anteriormente: velocidade do processador, memória $R A M$, espaço de armazenamento do $\mathrm{HD}$, assistência técnica autorizada. Ainda, a especificação sobre a memória cache foi acrescentada e estavam anteriormente presentes as informações sobre o processador e o sistema operacional. Todos estes atributos constavam no anúncio do produto no site da marca selecionada para experimentação.

$\mathrm{Na}$ condução propriamente dita do experimento, os participantes foram aleatoriamente divididos entre anúncio $\mathrm{A}$ e anúncio $\mathrm{B}$ e uma nova subdivisão, com efeito âncora e sem efeito âncora, que consistiu num vídeo com duração de 1 minuto e 27 segundos que apresentava os problemas causados pelo lixo eletrônico no planeta. $\mathrm{O}$ vídeo exposto é de autoria de Prado, Ulpiano e Cruz (2009), tendo sido adaptado para este experimento.

Todos os alunos presentes em sala de aula no momento da coleta que concordaram em participar da pesquisa foram os sujeitos. Após lerem e assinarem o Termo de Consentimento Livre e Esclarecido (TCLE) - a pesquisa foi submetida ao Comitê de Ética em Pesquisa da UFSM 
anteriormente a sua aplicação. O total de participantes do experimento foi: 170 alunos de graduação da UFSM (anúncio A com vídeo: 48; anúncio A sem vídeo: 40; anúncio B com vídeo: 43; anúncio B sem vídeo: 39). A Figura 2, acompanhada de legenda, apresenta os grupos experimentais de forma pormenorizada.

Segundo Siegel e Castellan Jr. (2006), a estatística do teste Mann-Whitney (U) é calculada tendo como parâmetro os tamanhos amostrais de cada grupo e a soma dos postos de um deles. Existe uma distribuição teórica e exata para a estatística U especialmente desenvolvida para amostras pequenas (número $\mathrm{n}<20$ ). Como a maioria dos grupos possui número menor que 20 (vinte), optou-se pela estatística não-paramétrica do referido teste.

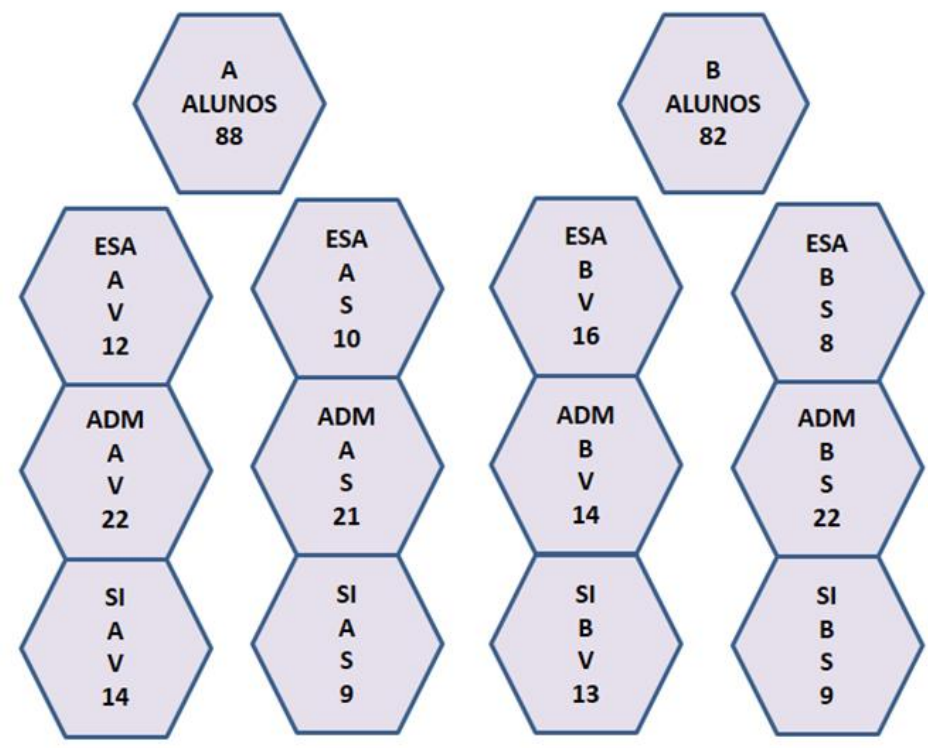

Figura 2 - Grupos experimentais da pesquisa Fonte: Elaborada pelos autores.

$\mathrm{Na}$ aplicação da tarefa, após instruções aos participantes e exibição do vídeo, os sujeitos tiveram que analisar o anúncio proposto. Definiu-se em distribuir um anúncio por respondente, pois caso o participante visualizasse os dois tipos ao mesmo tempo ele perceberia diferenciações entre eles, podendo levá-lo a entender a pista e enviesar suas respostas. Assim, tomou-se o extremo cuidado de que os respondentes do anúncio A não visualizassem o B. No total, obteve-se 4 grupos, entre experimentais e de laboratório.

Após a visualização do anúncio, foi distribuído para cada participante da pesquisa um questionário, a fim de mensurar a intenção de compra por meio de escala construída por Sujan (1985), com três níveis de sete pontos: negativa/positiva; ruim/boa; favorável/desfavorável, sendo o número 4 representante da posição neutra. Computa-se o resultado total de cada atitude por meio do somatório dos resultados dos três níveis de cada uma das três escalas. Assim, o escore das atitudes é uma medida que varia entre 3 e 21 .

Nesta etapa, além de marcar as alternativas, dentre as escalas apresentadas, os sujeitos tiveram que responder, de forma escrita, à pergunta: qual (is) aspecto (s) DO PRODUTO você mais levou em conta em sua opinião?

Antes da coleta dos dados foi lido o seguinte texto introdutório: supondo que você possui um crédito disponível de $\mathrm{R} \$ 2.500,00$ na Dell para compra de um notebook e que esteja interessado em utilizá-lo, você receba em seu e-mail o anúncio que lhe foi entregue de forma impressa. Estas orientações foram expostas a fim de criar uma situação fictícia que pudesse, de certa forma, neutralizar a pista preço e amenizar os efeitos da pista marca presentes no anúncio. 

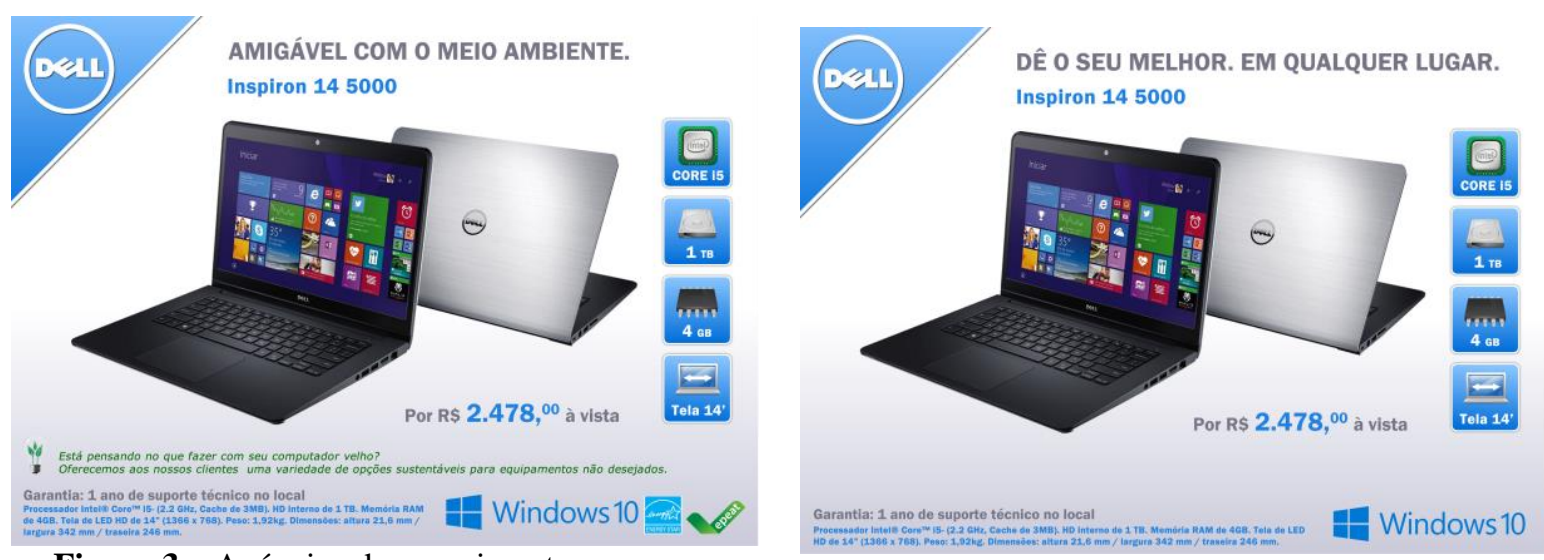

Figura 3 - Anúncios do experimento

Fonte: Elaborada pelos autores, com ajuda de designer gráfico.

Anúncio A (esquerda) $=$ experimental. Anúncio B $($ direita $)=$ de controle.

Verifica-se na Figura 3, que o primeiro anúncio (tratamento A) mostrou o produto com uma posição ecológica, com a inserção dos selos verdes da Epeat e Energy Star, mostrou-se como amigável com meio ambiente e apresentou um texto sobre opções para produtos não desejados. $\mathrm{O}$ segundo anúncio (tratamento B) não apresentou informação que tenha sido foco da pesquisa, sendo predominantemente técnico e com informação de preço.

\section{RESULTADOS DA PESQUISA}

Foram pesquisados 103 homens e 67 mulheres entre os respondentes. A média de idade foi de 23,79 anos, sendo predominantes os níveis de renda de $\mathrm{R} \$ 3.152,01$ a $\mathrm{R} \$ 7.880,00$ (com 33,53\%) e de $\mathrm{R} \$ 1.576,01$ a $\mathrm{R} \$ 3.152,00(30,59 \%)$.

No que diz respeito ao conhecimento dos sujeitos em relação à Epeat, apenas 5,29\% já ouviram falar desta ferramenta. Seguem as seções com as análises quantitativa e qualitativa da pesquisa.

\subsection{Análise quantitativa da pesquisa}

Os pesquisadores, usualmente, testam as hipóteses de que um grupo difere de outro em termos de atitudes, comportamento ou outra característica (Hair, Babin, Money \& Samouel, 2005). Nesse tópico, são apresentados os cruzamentos, a fim de verificar diferenças significativas entre os diferentes grupos, com base na estatística não-paramétrica, por meio do teste U de Mann Whitney.

Percebe-se na Tabela 1 que não existem diferenças significativas nas relações expostas ao se considerar o grupo de acadêmicos no geral, sendo $\mathrm{V}$ os alunos que assistiram ao vídeo e $\mathrm{S}$ os que não assistiram.

Tabela 1 - p-valores para os testes U de Man-Whitney - anúncios A e B - intenção de compra em relação à ancoragem - cursos de Engenharia Sanitária e Ambiental, Administração e Sistemas da Informação

\begin{tabular}{|c|c|c|c|c|c|c|}
\hline \multirow{3}{*}{ CONSTRUTO } & \multicolumn{3}{|c|}{ ANÚNCIO A } & \multicolumn{3}{|c|}{ ANÚNCIO B } \\
\hline & \multicolumn{2}{|c|}{ Mediana / Média } & \multirow[t]{2}{*}{ p-valor } & \multicolumn{2}{|c|}{ Mediana / Média } & \multirow[t]{2}{*}{ p-valor } \\
\hline & $\mathrm{V}$ & $S$ & & $\mathrm{~V}$ & $S$ & \\
\hline Intenção de compra & $16,5 / 15,42$ & $15 / 14,63$ & 0,2324 & $15 / 14,60$ & $16 / 15,72$ & 0,3802 \\
\hline
\end{tabular}

Porém, ao comparar os cursos (Engenharia Sanitária e Ambiental com vídeo - ESA V versus Engenharia Sanitária e Ambiental sem vídeo - ESA S; Administração com vídeo - ADM V versus 
Administração sem vídeo - ADM S; Sistemas da Informação com vídeo - SI V versus Sistemas da Informação sem vídeo - SI S), encontra-se diferença no curso de ESA. A Tabela 2 destaca os resultados que apresentaram diferença em nível de significância de 5\% (*) quando os cursos são emparelhados. Assim, na mencionada Tabela (2), nos grupos do curso de Engenharia Sanitária e Ambiental (ESA) que analisaram o anúncio B (sem AE), percebe-se que é encontrada diferença significativa em nível de 5\% de significância no que diz respeito à intenção de compra, sendo maior no grupo que não assistiu ao vídeo.

Tabela 2 - p-valores para os testes U de Man-Whitney para ancoragem - emparelhamento dos cursos em relação à intenção de compra - resultados significativos

\begin{tabular}{|c|c|c|c|}
\hline \multirow{3}{*}{$\begin{array}{c}\text { CONSTRUTOS } \\
\text { ATITUDES }\end{array}$} & \multicolumn{3}{|c|}{ ESA ANÚNCIO B } \\
\hline & Mediana & Média & p-valor \\
\hline & $\mathrm{V}$ & $\mathrm{S}$ & \\
\hline Intenção de compra & $14,5 / 13,50$ & $18,5 / 17$ & $0,0402 *$ \\
\hline
\end{tabular}

Fonte: Elaborada pelos autores

$\mathrm{A}=$ com argumento ecológico. $\mathrm{B}=$ sem argumento ecológico. $\mathrm{V}=$ exibição do vídeo. $\mathrm{S}=$ sem exibição do vídeo.

Ressalta-se que diferenças estatisticamente significativas não são encontradas em relação à ancoragem nos cursos de Administração e de Sistemas da Informação, levando em conta os dois tipos de anúncios, salientando que este grupo de alunos concentrou suas considerações em relação aos aspectos técnicos e de custo-benefício do aparelho. Supõe-se, então, que o emparelhamento dos cursos obteve o efeito pretendido.

Estas evidências podem indicar que conhecimento anterior e comportamento passado podem ser influenciadores do processo de ancoragem. Estes resultados vão ao encontro dos achados sobre ancoragem de Strack, Bahník e Mussweiler (2016), os quais concluíram que a acessibilidade e a aplicabilidade do conhecimento influenciam nos efeitos do julgamento do sujeito decisor. Epley e Gilovich (2006) ressaltam que ainda não está claro porque os ajustes tendem a ser insuficientes, constituindo-se numa lacuna significativa. Esta pesquisa supõe que a ancoragem depende de outros fatores, tais como interesse pelo assunto, área de formação/atuação, conhecimento.

\subsection{Análise qualitativa da pesquisa}

Apresenta-se um resumo da análise em relação à pergunta dissertativa que integrou o instrumento de coleta dos dados: qual(is) aspecto (s) DO PRODUTO você mais levou em conta em sua opinião? Os atributos foram organizados de forma decrescente por frequência de citação, considerando cinco posições, contando-se o quantitativo de pessoas que consideraram determinado aspecto do produto em sua opinião, desconsiderando a ordem de preferências individuais.

A Tabela 3 exibe os resultados referentes à ancoragem em relação ao anúncio A (com AE).

Tabela 3 - Aspectos do produto mais levados em conta - anúncio A - cursos de Engenharia Sanitária e Ambiental, Administração e Sistemas da Informação

\begin{tabular}{cccc}
\hline $\begin{array}{c}\text { Anúncio com argumento } \\
\text { ecológico - com âncora }\end{array}$ & Pessoas & $\begin{array}{c}\text { Anúncio com argumento } \\
\text { ecológico - sem âncora }\end{array}$ & Pessoas \\
\hline Configurações & 22 & Preço & 21 \\
Argumento ecológico - AE & 21 & Memória & 20 \\
Processador & 15 & Processador & 13 \\
Preço & 15 & Marca & 13 \\
Marca & 14 & Argumento ecológico - AE & 10 \\
\hline
\end{tabular}

Fonte: Elaborada pelos autores.

É possível verificar na Tabela 3 que, no grupo que houve a ancoragem do vídeo, o aspecto argumento ecológico figurou no segundo lugar entre os itens mais citados, representando 41,18\% 
das 51 respostas. Em contrapartida, no grupo que não ocorreu a ancoragem, o argumento ecológico aparece em quinto lugar como item mais frequente, representando 22,22\% do total das 45 respostas.

A análise qualitativa faz com que esta pesquisa transcenda os dados quantitativos, indo além dos números. Estes dados sugerem que boa parte dos respondentes considerou e referenciou o argumento ecológico nos aspectos do produto, principalmente quando sofreram a ancoragem.

\section{ANÁLISE E DISCUSSÃO DOS RESULTADOS}

De forma geral, as pessoas ancoraram, mas não houve influência na hora de decidir positivamente sobre um produto verde (em comparação com um produto não verde). Ou seja, verificou-se mais um discurso sustentável (análise qualitativa) do que um comportamento efetivo de compra de TI Verde (análise quantitativa).

Os cursos foram selecionados como parte do procedimento de emparelhamento sugerido por Vieira (1999). Os alunos, no geral, não apresentaram diferenças estatisticamente significativas em relação à intenção de compra considerando a ancoragem. Porém, no emparelhamento dos cursos, Engenharia Sanitária e Ambiental (ESA) apresentou forte evidência (diferença significativa em nível de significância de 5\%) de que, no grupo em que houve a exibição do vídeo, ocorreu diminuição da intenção de compra em relação ao anúncio B. Nesta análise quantitativa, os cursos de Administração (ADM) e de Sistemas da Informação (SI) não foram afetados pela ancoragem. Supõe-se que estes resultados são devidos à acessibilidade do conhecimento, ou seja, os alunos do curso de ESA estariam com o enfoque mais direcionado à sustentabilidade ambiental que os acadêmicos dos outros dois cursos.

Pesquisas empíricas demonstram que a magnitude de um efeito de ancoragem é independente da expertise dos julgadores, embora o conhecimento de algum assunto pudesse em alguns casos diminuir tal efeito (Schuery, 2012). Neste estudo, a ancoragem foi responsável por diminuir a intenção de compra dos alunos do curso de ESA (considerados aqui como conhecedores de assuntos de sustentabilidade ambiental) em relação ao anúncio sem o AE, ou seja, a utilização do vídeo foi responsável por efetuar diferenças de intenção de compra com a manipulação dos anúncios (com AE versus sem AE) neste grupo de alunos.

A análise qualitativa procurou verificar os aspectos do produto mais levados em conta na avaliação dos respondentes, suportando a análise quantitativa. Observou-se que o vídeo foi capaz de produzir a ancoragem, elevando consideravelmente o percentual de citação do argumento ecológico no anúncio A.

A união dos resultados quantitativos e qualitativos em relação à percepção da pista (argumento ecológico) alerta para a necessidade de uma comunicação mais eficiente por parte dos fabricantes, visando a uma maior conscientização do consumidor; prestando esclarecimentos sobre o que vem a ser um produto ecológico. É preciso realizar programas de sensibilização, campanhas, bem como os canais reversos devem ser bem comunicados aos consumidores.

Os resultados sugerem ainda a necessidade de maior inserção da Epeat no Brasil, já que apenas 5,29\% dos respondentes ouviram falar desta ferramenta. Verifica-se que esta se encontra inserida em nível internacional, carecendo de sua divulgação nacionalmente. As empresas precisam comunicar aos consumidores os produtos que possuem classificação bronze, prata ou ouro com destaque em suas propagandas de venda.

Em sua pesquisa, Pereira (2003) referencia a falta de informação sobre o que se constitui um produto ecologicamente correto. Há necessidade de esclarecimentos à população sobre a sustentabilidade ambiental aliada ao consumo. Para Chou (2013), entre os riscos para o reconhecimento da importância da TI Verde, estão a falta de conhecimento referente a esta temática e à sustentabilidade ambiental, falta de mudança comportamental e de responsabilidade social.

Verificou-se no site de comercialização de notebooks da marca utilizada nos anúncios que esta não dispõe de informações verdes diretamente relacionadas aos produtos, mas sim, em outras seções específicas do portal. Seria interessante se as empresas apresentassem as informações 
sustentáveis diretamente nas seções de venda. Para Melville (2010), o papel da informação em moldar atitudes é essencial. Desta forma, recomenda-se delinear claramente os atributos ambientais de computadores em comerciais e descrições dos produtos.

Em resumo, os resultados deste estudo indicam mais um discurso sustentável do que um comportamento efetivo de intenção de compra: na análise qualitativa, os alunos, no geral, mencionaram fortemente o argumento ecológico como aspecto do anúncio mais levado em conta na avaliação do produto (com ancoragem). Porém, na análise quantitativa, resultados estatisticamente relevantes não são encontrados. Entretanto, levando-se em conta o emparelhamento dos cursos, os resultados indicam que o efeito de ajuste do processo de ancoragem pode não ocorrer com todos os indivíduos, dependendo do contexto da aplicação: nível de conhecimento, interesse pelo assunto, área de atuação.

Por fim, a hipótese do trabalho - no anúncio com argumento ecológico, os sujeitos submetidos ao efeito âncora apresentam maior intenção de compra (Ic) - não foi aceita no presente experimento, sendo necessária a realização de mais estudos que considerem diferentes contextos de aplicação, tendo em vista os resultados apresentados na análise qualitativa e nos grupos pormenorizados do experimento.

\section{CONSIDERAÇÕES FINAIS}

Este estudo buscou verificar o efeito ancoragem na intenção de compra (Ic) dos consumidores de Tecnologia da Informação Verde. Os alunos, no geral, não apresentaram diferenças estatisticamente significativas em relação à Ic no anúncio com argumento ecológico, considerando a ancoragem. Ou seja, a hipótese da pesquisa não foi aceita.

Porém, ao comparar os cursos, a Engenharia Sanitária e Ambiental (ESA) apresentou forte evidência (diferença significativa com nível de significância de 5\%) de que no grupo em que houve a exibição do vídeo ocorreu redução da intenção de compra em relação ao anúncio B. Os cursos de Administração e de Sistemas da Informação (SI) não foram afetados pela ancoragem. Supõe-se que estes resultados são devidos à acessibilidade do conhecimento.

A análise qualitativa procurou verificar os aspectos do produto mais levados em conta na avaliação dos respondentes que analisaram o anúncio A (com AE). Observou-se que o vídeo foi capaz de produzir a ancoragem, sendo que elevou consideravelmente o percentual de citação do argumento ecológico. Assim, os resultados indicam mais um discurso sustentável do que um comportamento efetivo de intenção de compra. Ou seja, o argumento ecológico sensibiliza, mas não é capaz de influenciar a intenção de compra de forma efetiva.

Com este estudo, espera-se contribuir para uma maior inserção do consumo de TI Verde, com implicações acadêmicas, governamentais, mercadológicas e ambientais, especialmente: à sociedade, por meio da disseminação do conhecimento sobre consumo verde em relação aos produtos de TI e dos resultados deste experimento para uma mudança cultural gradativa e efetiva, por meio de um desenvolvimento sustentável.

No âmbito empresarial, os resultados desta pesquisa podem oferecer a oportunidade de desenvolver medidas concretas para comercialização de computadores orientados à TI Verde, campanhas publicitárias e promoções. De tal modo, na visão gerencial, este estudo poderá fornecer resultados para que empresas de TI possam aumentar a reputação corporativa. Porém, ressalta-se que o principal foco de análise é a dimensão relacionada à preservação do sistema ambiental, a qual as empresas possuem responsabilidades. Assim, a adoção destas práticas sustentáveis poderá melhorar o atual balanço ambiental negativo da TI, conforme Jayo e Valente (2010) sendo esta a pretensão principal deste trabalho em termos de implicações práticas.

À área de pesquisa sobre consumo consciente, especialmente em relação à experimentação, a fim de estimular estudos sobre esta temática, notadamente na área de TI Verde. Assim, espera-se ainda uma maior disseminação do tema, já que Schimidt et al. (2010) relatam não haver uma 
definição clara sobre TI Verde. Também há uma lacuna em relação aos ajustes insuficientes em relação à Heurística da Ancoragem e Ajustamento.

Algumas considerações sobre este estudo podem ser apontadas: a utilização de um design experimental traz limitações de validade externa. Além disso, no experimento, não se sabe até que ponto o atributo marca possa ter influenciado na avaliação dos sujeitos.

Desta forma, sugere-se agregar em futuras pesquisas a variável envolvimento com a marca (alto envolvimento versus baixo envolvimento), a fim de verificar a força desta na atitude dos consumidores de TI. Ainda, para investigações posteriores, sugere-se replicar este estudo em outros contextos. Também outros produtos de TI podem ser testados, tal como celulares. Apesar das limitações, espera-se que os resultados encorajem futuras pesquisas.

A agenda de TI Verde representa uma grande mudança nas prioridades para a indústria, profissionais, educadores, pesquisadores e usuários de tecnologia da informação, os quais devem estar preparados para ajustar seu nível de pensamento para perceber o potencial da TI (Murugesan, 2008). Assim, espera-se que esta pesquisa colabore para o aumento do poder da TI, no contexto de todos os agentes mencionados, para criar um ambiente sustentável em benefício das gerações atuais e futuras. De tal modo, a contribuição desta pesquisa está na introdução de ancoragem como uma heurística cognitiva para a área de consumo de TI Verde, a fim de estimular comportamentos sustentáveis.

\section{REFERÊNCIAS}

Bazerman, M. H. (2004). Processo decisório para cursos de administração e economia. Rio de Janeiro: Elsevier.

Berkhout, F., Hertin, J. (2001). Impacts of information and communication technologies on environmental sustainability: speculations and evidence. Recuperado em: 6 jun.2015, de <http://www.oecd.org/sti/inno/1897156.pdf>.

Bezerra, J. M. F., Leone, R J. G. (2013). Efeito ancoragem e relações de consumo: um estudo com produtos da cesta básica. Revista Ambiente Contábil, 5(2), 68 - 85.

Brunner, C. B., Ulrich, S., Jungen, P., Esch, F. (2016). Impact of symbolic product design on brand evaluations. Journal of Product \& Brand Management, 25(3), 307-320.

Caldeira, R. C. T. (2009). O impacto do argumento ecológico em atitudes relativas a um produto de alto envolvimento. Dissertação (Mestrado em Gestão Empresarial). Fundação Getúlio Vargas, Rio de Janeiro, RJ, Brasil.

Chou, D. C. (2013). Risk identification in Green IT practice. Computer Standards \&Interfaces, 35 , 231-237.

Cozby, P. C. (2006). Métodos de pesquisa em ciências do comportamento. São Paulo: Atlas.

Donoho, C. (2003) The "top-of-the-line" influence on the buyer-seller relationship. Journal of Business Research. Nova York, 56(10), 303-309.

Ebreo, A., Vining, J. (2001). How similar are recycling and waste reduction? Future orientation and reasons for reducing waste as predictors of self-reported behavior. Environment and Behavior, 33(3), 424-448. 
Elliot, S., Binney, D. (2008). Environmentally sustainable ICT: developing corporate capabilities and an industry-relevant IS Research Agenda. In: Pacis 2008 Proceedings. China. Energy Star (2016). About Energy Star. Recuperado em abril 2016, de: 〈https://www.energystar.gov/about>.

Engel, J.F., Blackwell, R.D.; Miniard, P.W. (2000). Comportamento do consumidor. Rio de Janeiro: LTC.

Epeat - Electronic Product Environmental Assessment Tool. (2016). Recuperado em: jan.2016, de: $<$ http://www.epeat.net/>.

Epley, N., Gilovich, T. (2006). The anchoring-and-adjustment heuristic. Why the adjustments are insufficient. Psychological Science, 17(4).

Esch, F. R. et al. (2009). The brand anchoring effect: a judgment bias resulting from brand awareness and temporary acessibility. Psychology \& Marketing, 26 (4), 383-395.

França, A. L. C., Milken, M. A., Teruel, R. L. H., Fagundes, A. F. A., Peixoto, F. M. (2017). Análise da heurística da ancoragem na precificação de automóveis seminovos em Uberlândia-MG. Revista de Administração da Unimep, 15 (2).

Gagarina, A., Pikturniene, I. (2015). The effect of ambient scent type and intensiveness on decision making heuristics. Procedia - Social and Behavioral Sciences, 213, 605 - 609.

Hair, J., Babin, B., Money, A. H., Samouel, P. (2005). Fundamentos de métodos de pesquisa em Administração. Porto Alegre: Bookman.

Jayo, M., Valente, R. (2010). Por uma TI mais Verde. GV Executivo, 9(1).

Koo, C., Chung, N. (2014). Examining the eco-technological knowledge of Smart Green IT adoption behavior: a self-determination perspective. Technological Forecasting \& Social Change, $88,140-155$.

Liu, Q. B; Karahanna, E. (2017). The dark side of reviews: the swaying effects of online product reviews on attribute preference construction. MIS Quarterly, 41 (2), 427-448.

Löbler, M. L. (2005). Processamento da informação: uma avaliação dos diferentes níveis de conhecimento no processo de decisão. Tese de Doutorado, Universidade Federal do Rio Grande do Sul, Porto Alegre, RS, Brasil.

Lopez, E. L., Silva, D., Hernandez, J. M. C. (2013). O efeito da marca na negligência da omissão: uma pesquisa experimental. $R A C$, Rio de Janeiro, 17(2), 132-153.

Luppe, M. R., Angelo, C. F. (2010). As decisões de consumo e a heurística da ancoragem: uma análise da racionalidade do processo de escolha. Rev. Adm. Mackenzie - RAM. São Paulo, 11(6), 81106.

Magalhães, S. (2013). Racionalidade limitada na tomada de decisão. O efeito de ancoragem na avaliação de Curriculum Vitae: um estudo experimental. Dissertação de mestrado, Universidade do Porto, Porto. 
Melville, N. P. (2010). Information systems innovation for environmental sustainability. MIS Quarterly, 34(1).

Murugesan, S. (2008). Harnessing green IT: principles and practices. IT Pro. Ieee Computer Society.

Mussweiler, T., Strack, F. (2001). The semantics of anchoring. Organizational Behavior and Human Decision Processes, 86, 234-255.

Pereira, S. J. N. (2003). O impacto do argumento ecológico nas atitudes dos consumidores: um estudo experimental. Dissertação de mestrado, Fundação Getúlio Vargas, Rio de Janeiro, RJ, Brasil.

Pereira, J. R. et al. (2015). Âncoras de decisão: um estudo de precificação com produtos originais e réplicas. Tourism \& Management Studies, 11(2), 189-196.

Prado, N. A. P., Ulpiano, J., Cruz, V. (2009). Lixo Eletrônico. Vídeo Educativo Pontifícia Universidade Católica (PUC), São Paulo - SP. Recuperado em: jan.2016, de:

<https://www.youtube.com/watch?v=NxwUmzdM_cc〉.

Reis, E. (2011). Prescrição ou descrição da decisão humana: uma discussão de um modelo subjacente ao SAD. Dissertação de Mestrado, Universidade Federal de Santa Maria, Santa Maria, RS, Brasil.

Russo, J. E., Schoemaker, P. J. (1993). Tomada de decisões: armadilhas. São Paulo: Saraiva.

Salles, A. C., Alves, A. P. F., Dolci, D. B., Lunardi, G. L. (2016). Tecnologia da informação verde: um estudo sobre sua adoção nas organizações. RAC. Rio de Janeiro, 20(1), 41-63.

Schimidt, N. H., Schmidtchen, T., Erek, K., Kolbe L., Zarnekow R. (2010). Influence of green IT on consumers' buying behavior of personal computers: implications from a conjoint analysis. 18th European Conference on Information Systems.

Schuery, J. M. (2012). Ancoragem e ajustamento e sua utilização em julgamento e tomada de decisão: um experimento no contexto brasileiro. Dissertação de Mestrado, Universidade Federal do Rio de Janeiro, Rio de Janeiro, Brasil.

Siegel, S., Castellan Jr., J. (2006). Estatística não-paramétrica para Ciências do Comportamento. Porto Alegre: Artmed.

Strack, F., Mussweiler, T. (1997). Explaining the enigmatic anchoring effect: mechanisms of selective accessibility. Journal of Personality and Social Psychology, 73 (3), 437-446.

Strack, F., Bahník, S., Mussweiler, T. (2016). Anchoring: accessibility as a cause of judgmental assimilation. Current Opinion in Psychology, 12, 67-70.

Sujan, M. (1985). Consumer knowledge: effects on evaluation strategies mediating consumer judgments. Journal of Consumer Research, 12, 31-45.

Techtudo (2013). Como comprar um notebook. Recuperado em: jan.2016, de:

$<$ http://www.techtudo.com.br/noticias/noticia/2013/10/confira-dicas-que-voce-precisa-saber-antesde-comprar-um-notebook.html>. 
Tversky, A., Kahneman, D. (1974). Judgment under uncertainty: heuristics and biases. Science, $185,1124-1131$.

Velter, A. N. et al. (2009). Atitudes dos consumidores a partir da teoria das pistas e da consciência ambiental: contribuições ao estudo do Green Marketing. Rev. Adm. UFSM. Santa Maria, 2 (3), 399416.

Vieira, S. (1999). Estatística experimental. São Paulo: Atlas.

Yadav, M. S. (1994). How buyers evaluate product bundles: a model of anchoring and adjustment. Journal of Consumer Research. 21(2), 342-353.

Wansink, B., Kent. R., Hoch, S. (1998). An anchoring and adjustment model of purchase quantity decisions. Journal of Marketing Research, 35(1), 71-81.

Data da submissão: 04/11/2017

Data de aceite: 07/03/2018 